\title{
Post-epidemic Era: Reflections and Suggestions on the Construction Path of Smart Cities
}

\author{
Tu Zhoujiao ${ }^{1, a}$ \\ ${ }^{1}$ Faculty of Public Administration, Southwest Jiaotong University \\ a1406912672@qq.com
}

\begin{abstract}
With the acceleration of economic globalization and urbanization, the normalization of epidemic situation means that China has formally entered the post epidemic era. Under the epidemic situation, the digital service of intelligent city is not only conducive to the prevention and control of the epidemic, but also beneficial to the resumption of work and production after the epidemic. The development of smart cities is in line with the development trend of future cities. However, through the lag of epidemic prevention and control, we can find that there are a series of problems in the construction of smart city, such as slow construction of information platform、 lack of information sharing mechanism and information-based talents. Therefore, this paper further puts forward two countermeasures and suggestions: unified planning and integrated utilization. Strengthen the operation of smart city to provide the wisdom of the urban development under the post epidemic era.
\end{abstract}

Keywords: Post-epidemic era, Smart city, Digital service, Information technology

\section{INTRODUCTION}

As early as at the Central Economic Work Conference in December 2018, the CPC Central Committee proposed to strengthen the construction of artificial intelligence, industrial Internet and other new infrastructure. In June 2020, Premier Li Keqiang of the State Council mentioned in the opening ceremony of China-ASEAN Digital Economy Cooperation Year that the current digital economy is of great significance for restoring the economic and social development of various countries [1].And smart city is an advanced form of urban informatization that makes full use of the new generation of information technology in various industries of the city. It usually uses the Internet and big data to enhance the effect of urban management and improve the quality of life of citizens [2].Smart city construction is in line with the trend of economic globalization and has become the main trend of future urban development.

After the outbreak in 2019,the national health committee general office issued a notice to emphasize the importance of strengthening data collection and analysis applications, developing telemedicine services, standardizing Internet diagnosis and treatment consulting services, deepening the Internet plus government service, and strengthening the foundation and safety guarantee.
At the same time, other departments also successively formulated corresponding policy and the epidemic form linkage mechanism, such as online library and special topic of "policies and measures to deal with the epidemic situation and support the development of enterprises" on the portal website of the Ministry of culture and tourism. In short, in the process of fighting COVID-19, big data centers and digital services, such as "health code", "place code" and "bus information registration form", have played an important role in helping China's epidemic prevention and control and resumption of work and production. It shows the wide application prospect and development potential of information service. But at the same time, the management of epidemic situation has also exposed the shortcomings of China's overall low level of digitization and the lack of smart city construction. Therefore, in the post-epidemic era, how will the emerging digital technology enable the continuous epidemic prevention and control, and provide more digital products and services for the construction of smart city? 


\section{APPLICATION OF WISDOM CITY DIGITAL SERVICES DURING THE EPIDEMIC PERIOD}

Compared with SARS in 2003, COVID-19 was more explosive, easy to transmit and widely spread. Therefore, the biggest characteristic to prevent and control the outbreak is the widely use of information technology means. It shows the delicacy and dynamic management of smart city to ensure the real "one game of the whole country" in epidemic prevention and control. The application of smart city digital services during the epidemic period mainly includes the following points: Firstly, the online epidemic prevention information collection means are diversified. "Health code", "license plate automatic recognition" and other means have been put forward, so as to ensure accurate identification of the health of personnel and the orderly flow of personnel. Secondly, online work and learning software is widely used. Online platforms represented by "Tencent Conference" and "DingDing" have helped resume work and schools and maintained economic operations. Thirdly, online consumption and entertainment platforms are active. As the prevention of the epidemic requires the elimination of close social contact, resulting in a rapid growth in takeaway food ordering, buying on errands, online video and other consumptions. It settles employment difficulties and other problems. Fourthly, networking and dynamic of epidemic information. The media platforms of local governments will update the progress of the epidemic situation every day, so as to make the information open and transparent and eliminate the panic feeling of the public [3].It can be seen that the application of big data in smart cities has brought great convenience and support for epidemic control and social governance, which played a role in promoting the development of people's livelihood, monitoring environmental quality and facilitating smart government affairs. The application of big data in the construction of smart cities is in line with the future development direction of cities [4].

Epidemic situation is like examination, which not only highlights the function and achievement of smart city, but also exposes the deficiency of smart city construction in the new environment. The normalization of epidemic situation not only means difficulties, but also represents development opportunities and innovation. Therefore, it is very important to clarify the characteristics of post epidemic era, reflect on the shortcomings of smart city construction and improve it.

\section{REFLECTIONS AND SUGGESTIONS ON THE CONSTRUCTION OF SMART CITY AFTER THE EPIDEMIC}

\subsection{Objectives and characteristics of intelligent city construction in the post-epidemic era}

In June 2020, Comrade Xi Jinping proposed the "post-epidemic era" for the first time, emphasizing the construction of a strong public health system and early warning system, and the use of "grid + big data" means to do a good job in long-term epidemic prevention [5].This indicates that China has officially entered the "post-epidemic era", and it is necessary to combine the "digital urban governance" and "digital epidemic prevention and control" in smart cities with epidemic prevention and control. It needs put more emphasis on the "Internet + government" and cloud information platform, so as to realize the "win-win" between epidemic prevention and smart city construction [6]. Therefore, the goal of smart city construction in the post epidemic era is "grid governance under information technology", which is characterized by the information sharing and resource exchange between cities.

\subsection{Difficulties in smart city construction during the post-epidemic era}

Although smart city provides the foundation for social governance digitalized transformation and provides information for COVID-19's prevention and control, it can also find a series of problems in the process of smart city construction. There are mainly as follows:

\subsubsection{Slow construction of information platform in wisdom city}

"Mask booking", "code scanning registration" and other government services with a large demand are often paralyzed due to the instability of some local government systems, which seriously affects the efficiency and effect of epidemic prevention and control and urban governance. Meanwhile, the third party exchange platform with huge flow of tiktok and micro-blog has caused false information and misleading public opinion because of lack of government and business officials' speeches. In addition, the infrastructure construction of urban informatization is not in place, and the level of urban informatization is low

\subsubsection{Information technology is not fully used}

The utilization rate of digital services such as "mobile phone code scanning" in the elderly, primary school students and other groups is not high. And due to the information construction gap between regions, intelligent life has not been fully popularized [7]. Based on this, many places use the "Online + offline" mode for grid 
investigation and detection. But the information collected manually is difficult to ensure its authenticity and accuracy, and it not only consume human resources, but also increase the cost of governance.

\subsubsection{No information sharing mechanism, Very little accurate tracking}

To establish a modern smart city to deal with the unexpected social problems, the most important thing is to establish a nationwide information sharing mechanism. During the epidemic prevention and control, not all public transport vehicles adopt real name system, and some cross regional and cross system data cannot be seamlessly connected. So, it is impossible to carry out active and accurate tracking. Due to the lack of information exchange mechanism between cities, and the untimely updating of information, grid governance is difficult to synchronize in different regions.

\subsubsection{Lack of informationized talents and imperfect training mechanism}

After the outbreak of COVID-19, the state set up a temporary emergency technology department to deal with unexpected information collection and detection problems. However, the temporary Technology Department has not received professional training and organizational training, so it is inevitable that there will be some problems in the actual operation process, such as uncoordinated organization and poor technology. This reduces the efficiency of government governance and information feedback, and makes epidemic prevention and control and social problems more difficult. Therefore, it is very necessary to cultivate a professional technical team in the case of normalization of epidemic situation.

\subsection{Suggestions on promoting the construction of smart cities in the post-epidemic era with high quality}

Based on the above analysis, the following four suggestions are proposed for the construction of smart cities in the post-epidemic era:

\subsubsection{Unified planning integrated utilization}

Smart city governance is to solve the complex problems of multi-level and multi-dimensional. Smart city construction should be planned from a long-term perspective. On the one hand, it is necessary to build a new integrated urban space infrastructure and a stable information platform; on the other hand, it is necessary to establish a "smart city group" of data sharing from the perspective of the whole country, expand the scope and frequency of information sharing among cities, and realize the real "national game".

\subsubsection{Integrated utilization}

The key of smart city lies in "wisdom". Wisdom means the use of various information technologies to integrate urban material resources, data resources and human resources to provide better digital products and services for urban residents. Specifically including the following points: first, set up more "service robots" and other urban service equipment to improve the quality of life; second, promote the opening and sharing of government data and enterprise data, and identify urban public data from the data mastered by relevant subjects as far as possible and incorporate them into the urban public data system. At the same time, we should standardize the data quality and data flow, and build a high-quality data governance system.

\subsubsection{Enhance the operation of a smart city.}

After the initial establishment of the smart city, the organization and operation is also very important, mainly including: first, the establishment of the city leadership organization system of up-down linkage, the cultivation of a professional technical and operation team, and the formation of vertical and horizontal cooperation mode; second, the strengthening of the "full cycle management" mode, the application of the "digital government" government services in the city "public governance", more concerned about people's livelihood and social issues Question. The whole process of the establishment, development, operation and maintenance of smart city is inseparable from citizens' opinions and government policies; third, combined with epidemic prevention and control and green ecology, we should cultivate new industry and new products to solve "urban disease" with the supply of intelligent scenes, and promote peopleoriented intelligent application.

\subsubsection{Establishment of urban early warning and feedback system}

The preliminary planning and operation of a city is not perfect, and all kinds of unexpected social events often occur, leading to the deviation of social operation from the expected goal. Therefore, it is necessary to establish a city crisis early warning system, through the collection of all kinds of effective information, predict the coming city problems, so as to take positive preventive measures. In addition, after the crisis, it is necessary to summarize the experience and feed it back to each organization department for adjustment, so as to deal with similar urban problems.

\section{CONCLUSION}

China has officially entered the post-epidemic era. The construction and development of smart cities cannot be separated from epidemic prevention and control and 
digital services. Therefore, "Internet + " has become a more core goal for the construction of smart cities. Only by improving the top-level involvement, optimizing the smart platform, and strengthening the construction of the organization and leadership team, can smart cities make an important contribution in the event of major public emergencies such as the COVID-19 epidemic, and promote the modernization of national governance system and governance capacity.

\section{AUTHORS' CONTRIBUTIONS}

The theoretical contributions of this paper are as follows: firstly, this paper mainly from the perspective of information technology, thinking about the technical difficulties in the construction and development of smart city, which provides a new perspective for the research of smart city. Secondly, the existing literature mainly studies smart city from intelligent building and urban development, but less considering the characteristics of social development stage, and from the perspective of social governance to view the construction of smart city. Therefore, this paper combines the characteristics of post epidemic era, and provides research ideas for the development of smart city from the perspective of digital services.

The practical contributions of this paper are as follows: The combination of smart city development and epidemic prevention and control in this paper is in line with the concept of international urban development and innovation, and also reflects the main problems facing the international community. By improving the quality of information technology and digital services to promote the development of smart city and maintain social stability, it is conducive to improve the quality of life of residents and solve the problems of urban development and management.

\section{ACKNOWLEDGMENTS}

After continuous efforts and unremitting persistence, the paper was finally completed. Thank you to all the teachers in the college's learning. Thanks for the opportunity and help from AI Scholar academic platform.

\section{REFERENCES}

[1] Li Yu. New opportunities for digital economy development in the post epidemic era [J]. Financial science, 2020 (8): 102-110

[2] Wang jiesong. Research on the application of big data and Internet of things technology in smart city [J]. Wireless Internet technology, 2018,15 (3): 29-30

[3] Fu rongxiao. Smart city should be the field support of smart Governance [J]. National governance, 2020 (27): 40-42
[4] Lai Yulin. Research on the application of big data technology in smart city construction $[\mathrm{J}]$. China new communication, 2021,23 (1): 113-114

[5] Zhu Tingting, Liu Qiang, Xu Jingyu. Grid governance and practice of smart city in post epidemic era $[\mathrm{J}]$. Information and communication technology and policy, 2020 (11): 23-28

[6] Li Guoqing. Smart city boosts the transformation of urban governance. Social science journal, 2020-0430 (004)

[7] Fan Li. Research on the path of promoting smart governance in urban communities -- Based on the investigation of smart governance practice in Suzhou Industrial Park [D]. Suzhou University, 2018 